\title{
An Iterative Scheme for Learning Gravity Compensation in Flexible Robot Arms
}

\author{
A. DE LUCA, S. PANZIERI
}

Dipartimento di Informatica e Sistemistica, Università di Roma "La Sapienza"

Via Eudossiana 18, 00184 Roma, Italy

\begin{abstract}
Mimicking the case of rigid robot arms, the set-point regulation problem for manipulators with flexible links moving under gravity can be solved by either model-based compensation or PID control. The former cannot be applied if an unknown payload is present or when model parameters are poorly estimated, while the latter requires fine and lengthy tuning of gains in order to achieve good performance on the whole workspace. Moreover, no global convergence proof has been yet given for PID control of flexible robot arms. In this paper, a simple iterative scheme is proposed for generating exact gravity compensation at the desired set point, without the knowledge of rigid or flexible dynamic model terms. The control law starts with a PD action on the error at the joint level, updating at discrete instants an additional feedforward term. Global convergence of the scheme is proved under a mild condition on the proportional gain and a structural property on the arm stiffness, which is usually satisfied in practice. Experimental results are presented for a two-link robot with a flexible forearm moving on a tilted plane.
\end{abstract}

Keywords. Robot Control, Flexible Manipulators, Iterative Learning, Gravity Compensation.

\section{INTRODUCTION}

Regulation of multi-link flexible manipulators is often performed using linear feedback laws that exploit inherent physical properties of the system. In absence of gravity, it can be shown that a simple proportional derivative (PD) feedback of the joint position error is sufficient to asymptotically stabilize any arm configuration (De Luca and Siciliano, 1992a). This strategy is a straightforward generalization of the known result for rigid robot arms (Takegaki and Arimoto, 1981). In addition, a proper feedback from the deflection variables may improve the transient characteristics (Lee et al., 1988).

For rigid manipulators under gravity, the most direct approach to set-point regulation is to globally cancel the gravity terms and still apply PD control with positive definite gains (Takegaki and Arimoto, 1981). Under a mild condition on the proportional gain, this nonlinear control law has been simplified to constant gravity compensation, evaluated only at the desired configuration (Tomei, 1991a); a purely linear feedback law with a feedforward action is then obtained. In this case, the proportional gain should dominate the gradient of the gravity forces in the whole robot workspace.

When flexible components are present in the robotic structure, a similar strategy based on PD plus feedforward terms has been shown to asymp- totically stabilize also robots with elastic joints (Tomei, 1991b) and with flexible links (De Luca and Siciliano, 1992b). For the elastic joint case, feedback is closed around the motor variables while for the flexible link case only the joint (rigid) variables are used for control. This compensation of gravity terms works under a further structural assumption on the joint or on the arm stiffness, respectively.

In all cases, an exact knowledge of the gravity vector is required. This condition is difficult to be realized, e.g. for a robot picking up multiple unknown payloads, and would need anyway an identification procedure of the robot link parameters. As a result of inexact compensation, a steadystate error will be present with this type of control even for a simple point-to-point task. For an arm with flexible links, the nature of this error is twofold: first, a displacement is present at the joint level (as in the rigid case); second, when considering the arm tip position, a further displacement is introduced by the arm deflection. High-gain feedback reduces but does not eliminate these errors, exciting on the other hand unmodeled dynamic effects (viz. higher order deformation modes) and leading to longer transition times because of the low damped oscillations.

To compensate for gravity effects, another standard remedy that does not require knowledge of the model is the addition of an integral term to the 
linear control law; however, several problems arise with the design of a PID, partly due to the nonlinear nature of the robot. Typically, saturation will occurr during large transient phases and reset or anti-windup procedures have to be devised when starting far from the final position (Åström and Wittenmark, 1990). From a theoretical point of view, asymptotic stability of robot PID control has been proved only for rigid arms. Moreover, it holds locally around the desired configuration and requires complex inequalities among the proportional, derivative, and integral gains to be satisfied (Arimoto et al., 1984a; Khorrami and Özgüner, 1988). In practice, some of these drawbacks may be overcome by adding the integral action only near the final point, so that gross motion is performed with PD control, while fine positioning is achieved with PID. However, no formal proof of convergence has been given for this method.

In this paper, we consider the set-point regulation problem for flexible manipulators under gravity and propose a fast iterative scheme that builds up the required compensation at the final configuration, with a very limited knowledge about the robot gravity terms. A PD-based control law is applied iteratively at the joint level and the constant gravity feedforward is learned without an explicit introduction of the integral error term nor the use of high-gain feedback.

An easy to check sufficient condition is given for the convergence of the scheme to zero steady-state error, taking into account in the analysis robot nonlinearities as well as arm deflections. In analogy with (De Luca and et al., 1990), the arm stiffness should dominate the gravity effects, an assumption which is usually satisfied in real flexible arms.

Experimental results are reported for a two-link lightweight manipulator, with a flexible forearm, available at the Robotics Laboratory of our Department (De Luca et al., 1990). The arm has been tilted from the horizontal plane so to include gravity effects. We mention that a similar iterative learning scheme was already shown to be convergent in the case of multi-link rigid robots and tested by simulation in (De Luca and Panzieri, 1992c).

\section{PRELIMINARIES}

For a robot arm composed of a serial chain of links, some of which are flexible, the Lagrangian technique can be used to derive the dynamic equations of motion (Book, 1984), modeling the slender links as Euler-Bernoulli beams with proper boundary conditions. A linear model is in general sufficient to capture the dynamics of each flexible link, but the interplay of rigid body motion and flexible deflections in the multi-link case gives rise to a full nonlinear dynamic model.
A set of basis space functions is used for describing link deformation shapes, with associated generalized coordinates. Denoting by $\theta$ the $n$-vector of joint coordinates and by $\delta$ the $m$-vector of link deformation coordinates, the $(n+m)$-vector $q=(\theta, \delta)$ characterizes the arm configuration.

We suppose to include only bending deformations limited, for each link, to the plane of rigid motion. The closed-form dynamic equations of the arm can be written as $n+m$ second-order nonlinear differential equations in the general form

$$
B(q) \ddot{q}+h(q, \dot{q})+g(q)+\left[\begin{array}{c}
0 \\
K \delta+D \dot{\delta}
\end{array}\right]=\left[\begin{array}{l}
u \\
0
\end{array}\right] .
$$

In (1), the $(n+m) \times(n+m)$ positive definite symmetric inertia matrix $B$ depends in general on both joint (rigid) and link (flexible) coordinates, while the $(n+m)$-vector $h$ contains Coriolis and centrifugal forces, and the positive definite (diagonal) matrix $D$ describes modal damping of the links. Note that deformations are described in frames which are clamped at the joint actuator sides, implying that the control does not enter directly in the equations of motion of the flexible part (De Luca and Siciliano, 1991).

The two positional terms in (1) come from the gravitational potential energy $U_{g}$ and from the elastic one $U_{\delta}$. In view of the small deformation hypothesis, we have in terms of energy that

$$
U_{\delta}=\frac{1}{2} \delta^{\mathrm{T}} K \delta \leq U_{\delta, \max }<\infty
$$

where $K$ is the positive definite symmetric (diagonal) stiffness matrix associated with link elasticity. A direct consequence of (2) is a bound on the deformation vector

$$
\|\delta\| \leq \sqrt{\frac{2 U_{\delta, \max }}{\lambda_{\max }(K)}}
$$

in terms of the maximum eigenvalue of $K$. On the other hand, the $(n+m)$-vector of gravity forces $g=\left(\partial U_{g} / \partial q\right)^{\mathrm{T}}$ can be partitioned as

$$
g(q)=\left(\begin{array}{c}
g_{\theta}(\theta, \delta) \\
g_{\delta}(\theta)
\end{array}\right)
$$

where the dependence of the lower term is justified by the assumed small deformations. Further, the vector $g$ satisfies the inequality

$$
\left\|\frac{\partial g}{\partial q}\right\| \leq \alpha_{0}+\alpha_{1}\|\delta\| \leq \alpha_{0}+\alpha_{1} \sqrt{\frac{2 U_{\delta, \max }}{\lambda_{\max }(K)}}=: \alpha
$$

where $\alpha_{0}, \alpha_{1}, \alpha>0$. Similarly, for the components of $(\partial g / \partial q)$ we have

$$
\left\|\frac{\partial g_{\theta}}{\partial q}\right\| \leq \alpha_{\theta}, \quad \quad\left\|\frac{\partial g_{\delta}}{\partial \theta}\right\| \leq \alpha_{\delta}
$$


with $\alpha_{\theta}, \alpha_{\delta}>0$. These bounds can be easily proven by observing that the gravity terms contain only trigonometric functions of $\theta$ and linear/trigonometric functions of $\delta$. As a direct consequence of (5) or (6), we have e.g.

$$
\left\|g\left(q_{1}\right)-g\left(q_{2}\right)\right\| \leq \alpha\left\|q_{1}-q_{2}\right\|, \quad \forall q_{1}, q_{2} \in \mathbb{R}^{n+m} .
$$

We remark that the above arguments and what follows can be easily modified to include also an explicit dependence of $g_{\delta}$ in (4) from $\delta$.

When the input torque $u$ is chosen as a PD control on the joint error

$$
u=K_{P}\left(\theta_{d}-\theta\right)-K_{D} \dot{\theta}, \quad K_{P}>0, K_{D}>0
$$

for a desired constant joint position $\theta_{d}$, then the robot arm will be driven to a steady-state condition $q=\bar{q}=(\bar{\theta}, \bar{\delta}), \dot{q}=0$, which from (1) satisfies the following equations

$$
\begin{aligned}
g_{\theta}(\bar{\theta}, \bar{\delta}) & =K_{P}\left(\theta_{d}-\bar{\theta}\right) \\
g_{\delta}(\bar{\theta}) & =-K \bar{\delta}
\end{aligned}
$$

implicitly defining the residual joint error $\theta_{d}-\bar{\theta}$ and the arm deformation $\bar{\delta}$.

Consider instead the joint PD+ control law, i.e.

$$
u=K_{P}\left(\theta_{d}-\theta\right)-K_{D} \dot{\theta}+g_{\theta}\left(\theta_{d}, \delta_{d}\right)
$$

with $K_{P}>0$ and $K_{D}>0$, being the associated $\delta_{d}$ defined by

$$
\delta_{d}=-K^{-1} g_{\delta}\left(\theta_{d}\right)
$$

It has been shown in (De Luca and Siciliano, 1992b) that, under the assumption

$$
\lambda_{\min }\left(\begin{array}{cc}
K_{P} & O \\
O & K
\end{array}\right)>\alpha
$$

$q=q_{d}=\left(\theta_{d}, \delta_{d}\right), \dot{q}=0$ is the unique equilibrium state of the closed-loop system, i.e. satisfying

$$
\begin{aligned}
g_{\theta}(\theta, \delta) & =K_{P}\left(\theta_{d}-\theta\right)+g_{\theta}\left(\theta_{d}, \delta_{d}\right), \\
g_{\delta}(\theta) & =-K \delta
\end{aligned}
$$

Condition (13) can always be satisfied, provided that an assumption on the structural link flexibility holds:

$$
\lambda_{\min }(K)=\min _{i=1, \ldots, n}\left\{k_{i}\right\}>\alpha
$$

being $K$ diagonal. In general this lower bound is not restrictive and depends on the relative magnitude of stiffness vs. gravity. As a result, by choosing the proportional control gain so that $\lambda_{\min }\left(K_{P}\right)>\alpha$, the equilibrium state $q=q_{d}$, $\dot{q}=0$ of system (1) under control (11) is asymptotically stable.
Similar considerations hold for an inexact constant gravity compensation $\left(\widehat{g}_{\theta}\right.$ in place of $\left.g_{\theta}\left(q_{d}\right)\right)$. Inequality (13) still guarantees a unique equilibrium configuration $\widehat{q}$, different from $q_{d}$.

\section{CONTROL SCHEME}

An iterative compensation scheme that achieves set-point regulation in a flexible robot, without knowledge of gravity, is introduced as follows. In particular, our objective is here to bring the vector of robot joint variables $\theta$ at a specified value $\theta_{d}$. Modifications for obtaining regulation of the arm tip position are straightforward.

Let $q_{0}=\left(\theta_{0}, \delta_{0}\right)$ be the initial arm configuration. The control law during iteration $i$ is defined as

$$
u=\frac{1}{\beta} K_{P}\left(\theta_{d}-\theta\right)-K_{D} \dot{\theta}+u_{i-1}, \quad \beta>0,
$$

for $i=1,2, \ldots$, where the term $u_{i-1}$ is a constant feedforward. If $u_{0}=0$, which is a common initialization, the first iteration is performed with a simple joint PD control. Indeed, one may collect the best available information on the required gravity term by setting $u_{0}=\widehat{g}_{\theta}\left(q_{d}\right)$, where the 'hat' denotes the estimate.

System (1) under control (17) reaches at the end of the $i$ th iteration the equilibrium state $q=q_{i}=$ $\left(\theta_{i}, \delta_{i}\right), \dot{q}=0$, such that

$$
\begin{aligned}
g_{\theta}\left(\theta_{i}, \delta_{i}\right) & =\frac{1}{\beta} K_{P}\left(\theta_{d}-\theta_{i}\right)+u_{i-1}, \\
g_{\delta}\left(\theta_{i}\right) & =-K \delta_{i} .
\end{aligned}
$$

Note that the unknown gravity term $g_{\theta}\left(q_{i}\right)$ is determined through the reading of the control effort at steady state. For the next iteration, the feedforward is istantaneously updated as

$$
u_{i}=\frac{1}{\beta} K_{P}\left(\theta_{d}-\theta_{i}\right)+u_{i-1},
$$

and control (17) is applied again starting from the current configuration $q_{i}$.

Our main result is the following:

Theorem. The sequence $\left\{\theta_{0}, \theta_{1}, \ldots\right\}$ converges to $\theta_{d}$, starting from any initial $q_{0}$, provided that:

$$
\begin{aligned}
& \lambda_{\min }(K)>\alpha \\
& \lambda_{\min }\left(K_{P}\right)>\alpha \\
& 0<\beta \leq \frac{1}{2} \frac{\alpha}{\alpha_{\theta}\left(1+\frac{\alpha_{\delta}}{\alpha}\right)} .
\end{aligned}
$$

Proof. Let $e_{i}=\theta_{d}-\theta_{i}$. At the end of the $i$ th iteration, eqs. (18) and (20) imply that $u_{i}=g_{\theta}\left(q_{i}\right)$ at the steady state $q_{i}$, and so

$$
\begin{aligned}
\left\|u_{i}-u_{i-1}\right\| & =\left\|g_{\theta}\left(q_{i}\right)-g_{\theta}\left(q_{i-1}\right)\right\| \\
& \leq \alpha_{\theta}\left\|q_{i}-q_{i-1}\right\| \\
& \leq \alpha_{\theta}\left(\left\|\theta_{i}-\theta_{i-1}\right\|+\left\|\delta_{i}-\delta_{i-1}\right\|\right)
\end{aligned}
$$


where the first inequality (6) was used. From eq. (19), using the second inequality in (6) and hypothesis (a), we have

$$
\begin{aligned}
\left\|\delta_{i}-\delta_{i-1}\right\| & \leq\left\|K^{-1}\right\| \cdot\left\|g_{\delta}\left(\theta_{i}\right)-g_{\delta}\left(\theta_{i-1}\right)\right\| \\
& <\frac{1}{\alpha} \alpha_{\delta}\left\|\theta_{i}-\theta_{i-1}\right\| .
\end{aligned}
$$

Combining (21) and (22),

$$
\begin{aligned}
\left\|u_{i}-u_{i-1}\right\| & <\alpha_{\theta}\left(1+\frac{\alpha_{\delta}}{\alpha}\right)\left\|\theta_{i}-\theta_{i-1}\right\| \\
& \leq \alpha_{\theta}\left(1+\frac{\alpha_{\delta}}{\alpha}\right)\left(\left\|e_{i}\right\|+\left\|e_{i-1}\right\|\right) .
\end{aligned}
$$

On the other hand, from eq. (20)

$$
\left\|u_{i}-u_{i-1}\right\|=\frac{1}{\beta}\left\|K_{P} e_{i}\right\|
$$

From eqs.(23) and (24), using hypothesis (b), it follows

$$
\begin{aligned}
\frac{1}{\beta} \alpha\left\|e_{i}\right\| & <\frac{1}{\beta} \lambda_{\min }\left(K_{P}\right)\left\|e_{i}\right\| \leq \frac{1}{\beta}\left\|K_{P} e_{i}\right\| \\
& <\alpha_{\theta}\left(1+\frac{\alpha_{\delta}}{\alpha}\right)\left(\left\|e_{i}\right\|+\left\|e_{i-1}\right\|\right) .
\end{aligned}
$$

Reorganizing terms, since hypothesis (c) implies $\alpha-\beta \alpha_{\theta}\left(1+\frac{\alpha_{\delta}}{\alpha}\right)>0$, we obtain

$$
\left\|e_{i}\right\|<\frac{\beta \alpha_{\theta}\left(1+\frac{\alpha_{\delta}}{\alpha}\right)}{\alpha-\beta \alpha_{\theta}\left(1+\frac{\alpha_{\delta}}{\alpha}\right)}\left\|e_{i-1}\right\| .
$$

Therefore, the error norm in (26) satifies a contraction mapping condition if

$$
\frac{\beta \alpha_{\theta}\left(1+\frac{\alpha_{\delta}}{\alpha}\right)}{\alpha-\beta \alpha_{\theta}\left(1+\frac{\alpha_{\delta}}{\alpha}\right)} \leq 1,
$$

which is again guaranteed by hypothesis (c). As a result, $\lim _{i \rightarrow \infty}\left\|e_{i}\right\|=0$, and asymptotic convergence of $\left\{\theta_{i}\right\}$ to $\theta_{d}$ is proved for any initial arm configuration $q_{0}$.

Q.E.D.

Hypotheses (a) and (b) are the same given in (De Luca and Siciliano, 1992b) for showing that the joint PD control law with constant known gravity compensation is globally asymptotically stable. In the present case, they are needed to assure that the robot arm under control (17) has a unique steady-state solution at every iteration. The new hypothesis (c) guarantees the convergence of the iterative scheme (20), and in particular that $\lim _{i \rightarrow \infty} u_{i}=g_{\theta}\left(q_{d}\right)$, with a priori knowledge limited to the bounds (5) and (6) on the gravity terms.

A series of remarks are now in order:

- The same proof can be followed in the case of rigid robot arms. In that case, $\alpha_{\delta}=0$, $\alpha_{\theta}=\alpha$, and it follows that $\beta \leq \frac{1}{2}$ (De Luca and Panzieri, 1992c). Merging conditions (b) and (c) into (17), the overall proportional gain matrix $\hat{K}_{P}=K_{P} / \beta$ has to be chosen so to satisfy

$$
\lambda_{\min }\left(\hat{K}_{P}\right)>2 \alpha
$$

- The iterative scheme (17) and (20) is reminiscent of learning control algorithms that achieve reproduction of repetitive trajectories for rigid (Arimoto et al., 1984a; De Luca et al., 1992d) or flexible robot arms (Poloni and Ulivi, 1991). However, no repositioning of the arm into the initial configuration is performed (nor required) here, at any iteration.

- The overall scheme can be interpreted as a discrete-time PID, in which the integral term is updated only at fixed instants. Moreover, this approach combines in an automatic way the benefits of a PD control far from the destination and of an integral action close to the goal, avoiding wind-up effects. As a further merit of the scheme, one should consider that gains with guaranteed convergence properties are easily selected.

- The bounds (5) and (6) on the gravity terms may be evaluated taking into account the maximum admissible payload, so to ensure exact set-point regulation in all operating conditions. Moreover, they can be directly obtained through experimental trials.

- As a drawback, since each update of the feedforward term should be performed at steady-state, the control scheme converges to the desired position in double infinite time. However, ultimate boundedness of the error in finite time is obtained by updating the feedforward term as soon as joint variations definitely drop below a given threshold, even before a complete stop.

- An interesting aspect is to estimate the distance from necessity of the sufficient conditions $(\mathrm{a}-\mathrm{c})$. This point can be investigated through simulations and experiments. In our experience, the above criteria are rather stringent.

\section{DESIGN FOR A TWO-LINK FLEXIBLE ROBOT}

The design of gains in the iterative control algorithm will be carried out for the two-link lightweight manipulator, with a flexible forearm, available at the Robotics Laboratory of our Department. 


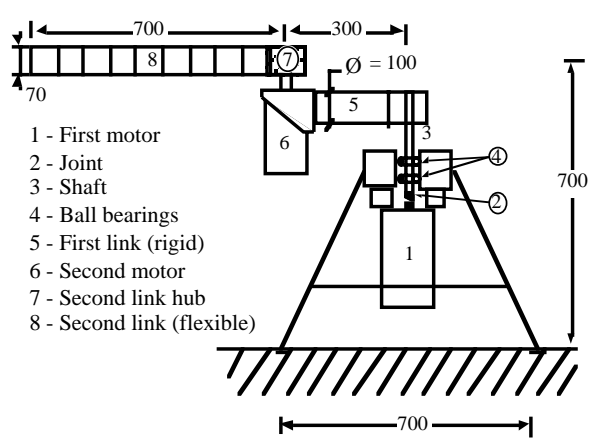

Fig. 1 - The two-link flexible robot arm at DIS

The robot arm is a planar mechanism constituted by two links, respectively $0.3 \mathrm{~m}$ and $0.7 \mathrm{~m}$ long, connected by revolute joints, and mounted on a fixed basement as shown in Fig. 1. The upper link is rigid while the second link, weighting $1.8 \mathrm{~kg}$, is very flexible in the plane of motion but relatively stiff with respect to bending in the orthogonal plane and torsion. Two d.c. motors are located at the joints in a direct-drive arrangement and deliver an actual peak torque of $7.2 \mathrm{Nm}$ and $3.8 \mathrm{Nm}$, respectively. Incremental encoders with 20000 pulses/turn and d.c. tachometers with 12bit D/A conversion are available for joint position and velocity feedback. To improve damping properties of arm dynamics, an analog velocity loop is directly closed at the power amplifier level around both joints. The forearm deformation is measured at three different points along the link, by means of an on-board optical transducer with $0.09^{\circ}$ of angular accuracy (Lucibello and Ulivi, 1989). The manipulator is interfaced with a $386 \mathrm{PC}$ control computer, allowing to execute simple control laws with sampling times of $5 \mathrm{msec}$.

A Lagrangian dynamic model of this flexible robot arm was derived in (De Luca et al., 1990). A modal analysis shows that two assumed modes are sufficient to capture the relevant flexibility of the second link, whose bending deflection $w$ is expressed as

$$
w(x, t)=\sum_{i=1}^{2} \phi_{i}(x) \delta_{i}(t) \quad i=1,2 .
$$

The following data characterize the arm:

$$
\begin{aligned}
\ell_{1} & =0.3 \mathrm{~m} & m_{h 2} & =3.1 \mathrm{~kg} \\
\ell_{2} & =0.7 \mathrm{~m} & m_{p} & =J_{p}=0 \\
J_{1 T o t} & =0.447 \mathrm{~kg} \mathrm{~m}^{2} & \phi_{1}\left(\ell_{2}\right) & =-1.446 \mathrm{~m} \\
J_{2 T o t} & =0.303 \mathrm{~kg} \mathrm{~m}^{2} & \phi_{2}\left(\ell_{2}\right) & =1.369 \mathrm{~m} \\
J_{h 2} & =6.35 \cdot 10^{-4} \mathrm{~kg} \mathrm{~m}^{2} & &
\end{aligned}
$$

where the subscript ' $h 2$ ' denotes the motor at the second hub. The first two natural frequencies of vibration are computed as:

$$
f_{1}=4.716 \mathrm{~Hz}, \quad f_{2}=14.395 \mathrm{~Hz} .
$$

The stiffness coefficients of the diagonal matrix $K$ are

$$
k_{1}=878.02 \mathrm{~N}, \quad k_{2}=8180.56 \mathrm{~N},
$$

while the diagonal damping matrix $D$ has elements:

$$
d_{1}=4.14 \mathrm{~N} \cdot \mathrm{sec}, \quad d_{2}=5.42 \mathrm{~N} \cdot \mathrm{sec} .
$$

Finally, the two following coefficients related to the mode shapes appear in the model:

$$
v_{1}=0.48 \mathrm{~kg} \cdot \mathrm{m}, \quad v_{2}=0.18 \mathrm{~kg} \cdot \mathrm{m},
$$

where

$$
v_{i}=\int_{0}^{\ell_{2}} \rho \phi_{i}(x) d x, \quad i=1,2 .
$$

In order to include gravity effects in our experiments, the manipulator base has been tilted by $\gamma \simeq 6^{\circ}$ from the horizontal plane. The associated model term $g(q)$ is reported below (standard abbreviations are used for sine and cosine):

$$
g_{\theta}=\left[\begin{array}{l}
g_{1} \\
g_{2}
\end{array}\right], \quad g_{\delta}=\left[\begin{array}{l}
g_{3} \\
g_{4}
\end{array}\right],
$$

with

$$
\begin{aligned}
& g_{1}=A_{1} s_{1}+A_{2} s_{12}+\left(A_{3} \delta_{1}+A_{4} \delta_{2}\right) c_{12} \\
& g_{2}=A_{2} s_{12}+\left(A_{3} \delta_{1}+A_{4} \delta_{2}\right) c_{12} \\
& g_{3}=A_{3} s_{12} \\
& g_{4}=A_{4} s_{12} .
\end{aligned}
$$

The constant coefficients are:

$$
\begin{aligned}
& A_{1}=g_{0}\left(m_{1} \ell_{c 1}+\left(m_{2}+m_{h 2}+m_{p}\right) \ell_{1}\right) \\
& A_{2}=g_{0}\left(m_{2} \ell_{c 2}+m_{p} \ell_{2}\right) \\
& A_{3}=g_{0}\left(m_{p} \phi_{1}\left(\ell_{2}\right)+v_{1}\right) \\
& A_{4}=g_{0}\left(m_{p} \phi_{2}\left(\ell_{2}\right)+v_{2}\right),
\end{aligned}
$$

being $g_{0}=9.8 \sin \gamma$ the actual gravity acceleration and $\ell_{c i}$ the distance from joint $i$ to the center of mass of link $i$. With this convention, $q=\left(\theta_{1}, \theta_{2}, \delta_{1}, \delta_{2}\right)=(0,0,0,0)$ corresponds to the straight downward position (of minimum potential energy). Note that $g_{\delta}$ is only a function of $\theta$, as anticipated.

For evaluating $\alpha$, the matrix $(\partial g / \partial q)$ can be readily computed. With the given data, a value $\alpha \simeq 2.85$ results, attained for $q=0$. The same value is used as an upper bound for $\alpha_{\theta}$ and $\alpha_{\delta}$.

\section{EXPERIMENTAL RESULTS}

In the first experiment, a motion from $q=0$ (undeformed arm) to the straight position of maximum gravity force ( $\pi / 2$ of first joint clockwise rotation) is commanded, using as proportional and derivative gains

$$
\begin{aligned}
& \hat{K}_{P}=\operatorname{diag}\{10.7,11.6\}, \\
& K_{D}=\operatorname{diag}\{1.6,0.85\} .
\end{aligned}
$$


The update (20) for $u_{i}$ is made at fixed intervals of 5 seconds. Figures $2-5$ show the joint errors and the applied torques over 14 seconds. In this case, two updates are sufficient for regulating the error to zero within 11 seconds. Note that both position gains in (39) satisfy the combined sufficient condition (b) and (c). The evolution of the tip deflection angle, as seen from the second link base, is given in Fig. 6, indicating that a maximum deflection of $\approx 0.7 \cdot 9 \cdot(\pi / 180)=10 \mathrm{~cm}$ is attained during motion while the residual tip deformation is $\approx 2 \mathrm{~cm}$.

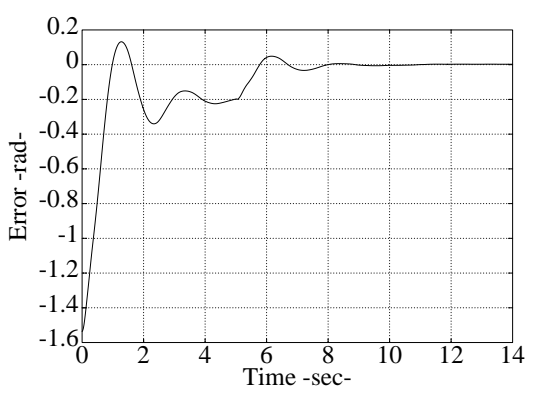

Fig. $2-$ Position error for joint $1\left(\theta_{d}=(-\pi / 2,0)\right)$

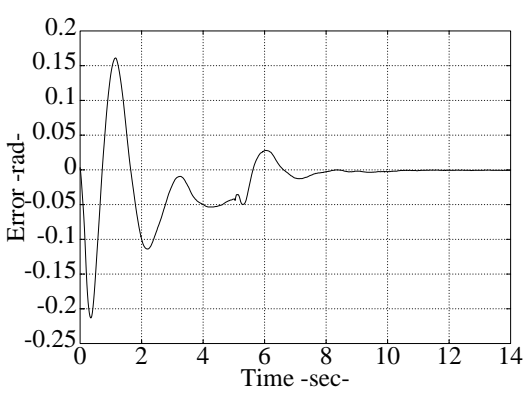

Fig. 3 - Position error for joint $2\left(\theta_{d}=(-\pi / 2,0)\right)$

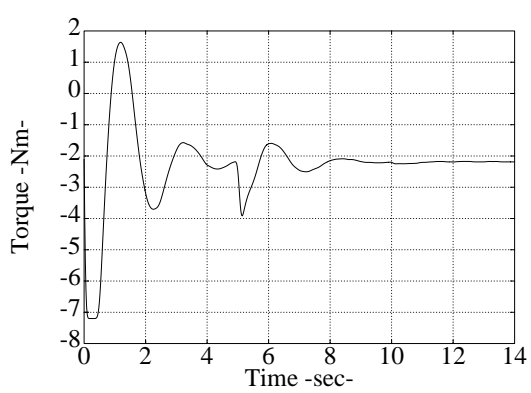

Fig. 4 - Applied torque for joint $1\left(\theta_{d}=(-\pi / 2,0)\right)$

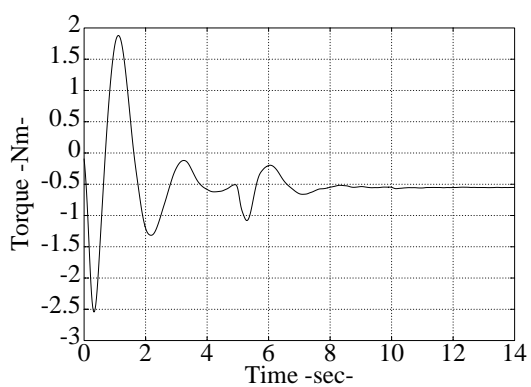

Fig. 5 - Applied torque for joint $2\left(\theta_{d}=(-\pi / 2,0)\right)$

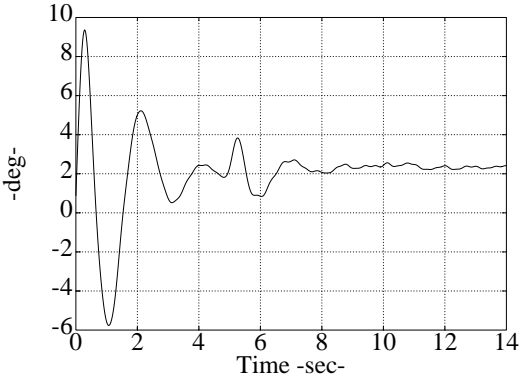

Fig. 6 - Tip deflection angle $\left(\theta_{d}=(-\pi / 2,0)\right)$

In the second experiment, $\theta_{d}=(-3 \pi / 4,0)$ is the desired joint position to be reached from the same initial configuration, using as gains

$$
\begin{aligned}
& \hat{K}_{P}=\operatorname{diag}\{5.7,6.2\}, \\
& K_{D}=\operatorname{diag}\{2.5,1.34\}
\end{aligned}
$$

Joint errors, input torques, and tip deflection angle over 25 seconds are displayed in Figs. 7-11. Four updates are now necessary for obtaining convergence. No special care was taken for minimizing the duration of the motion: a faster global transient could have been obtained by updating sooner the feedforward $u_{2}$, then $u_{3}$, and finally $u_{4}$. This example shows the capability of learning the exact gravity compensation also when the 'wall' of maximum gravity force has to be overcome. Notice that intermediate steady-state torques lie now on both sides of the final required values, indicating that the learning scheme is also able to reduce feedforward terms when needed. In fact, the control scheme was found to converge without problems also when resetting the desired set point back to the initial position $\theta=0$, where the required compensation is zero (an equilibrium point).

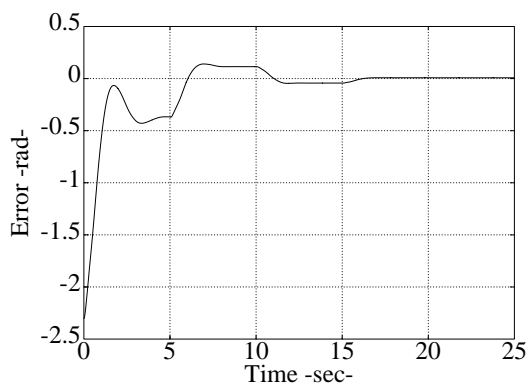

Fig. 7 - Position error for joint $1\left(\theta_{d}=(-3 \pi / 4,0)\right)$

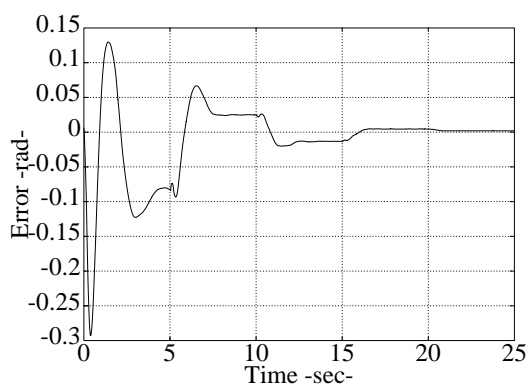

Fig. 8 - Position error for joint $2\left(\theta_{d}=(-3 \pi / 4,0)\right)$ 
control part is sufficient to prove global convergence of the scheme. Experimental results have shown the effectiveness of the approach, pointing out that the convergence condition is also close to be necessary.

The approach was implemented for the regulation of a desired joint configuration of the arm; in this respect, link deformation variables are not needed neither for feedback nor for the feedforward update. If the tip location is of interest, a similar learning scheme can be set up, still closing the feedback loop at the joint level but taking into account the value of link deformation at intermediate steady states for the update of the feedforward term.

\section{Acknowledgments}

This paper is based on work partly supported by Consiglio Nazionale delle Ricerche under contract 92.01115.PF67 (Progetto Finalizzato Robotica).

\section{REFERENCES}

Arimoto, S., S. Kawamura, and F. Miyazaki (1984a). "Bettering operation of robots by learning," J. of Robotic Systems, vol. 1, pp. $123-140$.

Arimoto, S., and F. Miyazaki (1984b). "Stability and robustness of PID feedback control for robot manipulators of sensory capability," in 1st Int. Symp. on Robotics Research, M. Brady and R.P. Paul (Eds.), MIT Press, Boston, MA, pp. 783-799.

Åström, K.J., and B. Wittenmark (1990). Computer-Controlled Systems: Theory and Design, 2nd Ed., Prentice-Hall International, London.

Book, W.J. (1984). "Recursive Lagrangian dynamics of flexible manipulator arms," Int. J. of Robotics Research, vol. 3, no. 3, pp. 87-101.

De Luca, A., L. Lanari, P. Lucibello, S. Panzieri, and G. Ulivi (1990). "Control experiments on a two-link robot with a flexible forearm," 29th IEEE Conf. on Decision and Control, Honolulu, HI, pp. 520-527.

De Luca, A., and B. Siciliano (1991). "Closedform dynamic model of planar multi-link lightweight robots," IEEE Trans. on Systems, Man, and Cybernetics, vol. 21, pp. 826-839.

De Luca, A., and B. Siciliano (1992a). "Relevance of dynamic models in analysis and synthesis of control laws for flexible manipulators," in Robotics and Flexible Manufacturing Systems, J.C. Gentina and S.G. Tzafestas (Eds.), Elsevier, Amsterdam, NL, pp. 161-168.

De Luca, A., and B. Siciliano (1992b). "An asymptotically stable joint PD controller for robot arms with flexible links under gravity," 31st IEEE Conf. on Decision and Control, Tucson, AZ, pp. 325-326.
De Luca, A., and S. Panzieri (1992c). "A simple iterative scheme for learning gravity compensation in robot arms," 36th ANIPLA Annual Conf. (Automation 1992), Genova, I, pp. 459471 .

De Luca, A., G. Paesano, and G. Ulivi (1992d) "A frequency-domain approach to learning control: Implementation for a robot manipulator," IEEE Trans. on Industrial Electronics, vol. 39, pp. $1-10$.

Khorrami, F., and Ü. Özgüner (1988). "Decentralized control of robot manipulators via state and proportional-integral feedback," 1988 IEEE Int. Conf. on Robotics and Automation, Philadelphia, PA, pp. 1198-1203.

Lee, H.G., S. Arimoto, and F. Miyazaki (1988). "Liapunov stability analysis for PDS control of flexible multi-link manipulators," 27th IEEE Conf. on Decision and Control, Austin, TX, pp. $75-80$.

Lucibello, P., and G. Ulivi (1989). "An optical angular transducer for flexible robot arms," Prep. 2nd IFAC Symp. on Low Cost Automation, Milano, I, pp. T113-T116.

Poloni, M., and G. Ulivi (1991). "Iterative learning control of a one-link flexible manipulator," 3rd IFAC Symp. on Robot Control, Vienna, A, pp. 393-398, 1991.

Takegaki, M., and S. Arimoto (1981). "A new feedback method for dynamic control of manipulators," Trans. ASME J. on Dynamic Systems, Measurement, and Control, vol. 102, no. 2, pp. 119-125.

Tomei, P. (1991a) . "Adaptive PD controller for robot manipulators," IEEE Trans. on Robotics and Automation, vol. 7, pp. 565-570.

Tomei, P. (1991b). "A simple PD controller for robots with elastic joints," IEEE Trans. on Automatic Control, vol. 36, pp. 1208-1213. 